\title{
A Novel ALDH5A1 Mutation Is Associated With Succinic Semialdehyde Dehydrogenase Deficiency and Severe Intellectual Disability in an Iranian Family
}

\section{Lucia Püttmann, ${ }^{1,2}$ Henning Stehr, ${ }^{3}$ Masoud Garshasbi, ${ }^{1}$ Hao Hu, ${ }^{1}$ Kimia Kahrizi, ${ }^{4}$ Bettina Lipkowitz, ${ }^{1}$ Payman Jamali, ${ }^{5}$ Andreas Tzschach, ${ }^{1}$ Hossein Najmabadi, ${ }^{4}$ Hans-Hilger Ropers, ${ }^{1}$ Luciana Musante, ${ }^{1 *}$ and Andreas W. Kuss ${ }^{1,6 *}$}

\author{
${ }^{1}$ Department of Human Molecular Genetics, Max Planck Institute for Molecular Genetics, Berlin, Germany \\ ${ }^{2}$ Department of Biology, Chemistry and Pharmacy, Freie Universität Berlin, Berlin, Germany \\ ${ }^{3}$ Department of Vertebrate Genomics, Max Planck Institute for Molecular Genetics, Berlin, Germany \\ ${ }^{4}$ Genetics Research Center, University of Social Welfare and Rehabilitation Sciences, Tehran, Iran \\ ${ }^{5}$ Shahroud Welfare Organization, Semnan, Iran \\ ${ }^{6}$ Institute for Human Genetics, University Medicine Greifswald \& Interfaculty Institute for Genetics and Functional Genomics, \\ Ernst Moritz Arndt University, Greifswald, Germany
}

Manuscript Received: 18 July 2012; Manuscript Accepted: 15 April 2013

\begin{abstract}
Succinic semialdehyde dehydrogenase (SSADH) deficiency is a disorder of the catabolism of the neurotransmitter gammaaminobutyric acid (GABA) with a very variable clinical phenotype ranging from mild intellectual disability to severe neurological defects. We report here on a large Iranian family with four affected patients presenting with severe intellectual disability, developmental delay and generalized tonic-clonic seizures. Molecular genetic analysis revealed a missense mutation c.901A > G (p.K301E, RefSeq number NM_001080) in ALDH5A1 co-segregating with the disease in the family. The missense mutation affects an amino acid residue that is highly conserved across the animal kingdom. Protein modeling showed that p.K301E most likely leads to a loss of $\mathrm{NAD}^{+}$binding and a predicted decrease in the free energy by $6.67 \mathrm{kcal} / \mathrm{mol}$ furthermore suggests a severe destabilization of the protein. In line with these in silico observations, no SSADH enzyme activity could be detected in patient lymphoblasts. ๑ 2013 Wiley Periodicals, Inc.
\end{abstract}

Key words: SSADH deficiency; ALDH5A1; intellectual disability; Iranian; autosomal recessive

\section{INTRODUCTION}

Succinic semialdehyde dehydrogenase (SSADH) deficiency (OMIM \#271980) is a rare autosomal recessive inherited metabolic disorder resulting in the accumulation of $\gamma$-hydroxybutyrate (GHB) in the brain [Mason and Kerns, 2002; Pearl et al., 2003]. In patients with SSADH deficiency, brain concentrations of GHB are increased 30 -fold and concentrations of $\gamma$-amino-butyric acid (GABA) are increased two- to fourfold as compared to healthy
How to Cite this Article:

Püttmann L, Stehr H, Garshasbi M, Hu H, Kahrizi K, Lipkowitz B, Jamali P, Tzschach A, Najmabadi H, Hilger H, Musante L, Kuss AW. 2013. A novel ALDH5A1 mutation is associated with succinic semialdehyde dehydrogenase deficiency and severe intellectual disability in an Iranian family.

Am J Med Genet Part A 161A:1915-1922.

\section{Conflict of interest: None}

Masoud Garshasbi's present address is Faculty of Medical Sciences, Department of Medical Genetics, Tarbiat Modares University, Tehran, Iran

Grant sponsor: Iranian National Science Foundation and the Max Planck Innovation Fund.

*Correspondence to:

Luciana Musante, Department of Human Molecular Genetics, Max Planck Institute for Molecular Genetics, Ihnestr. 73, 14195 Berlin, Germany, and Andreas W. Kuss, Institute for Human Genetics, University Medicine Greifswald \& Interfaculty Institute for Genetics and Functional Genomics, Ernst Moritz Arndt University, Greifswald, Germany.

E-mail: musante@molgen.mpg.de (L.M.), kussa@uni-greifswald.de (A.W.K.)

Article first published online in Wiley Online Library

(wileyonlinelibrary.com): 4 July 2013

DOI 10.1002/ajmg.a.36030 
individuals [Gibson et al., 1998]. Concordant with this phenotype, SSADH deficient mice ( $A L D H 5 A 1^{-/-}$) show a 60 -fold increase of GHB and a 2-fold increase of GABA levels in the brain [Hogema et al., 2001; Jansen et al., 2008].

The major inhibitory neurotransmitter of the brain, GABA, is derived from the major excitatory neurotransmitter glutamate. GABA is in turn converted into succinic semialdehyde that is then oxidized to succinate by the $\mathrm{NAD}^{+}$-dependent succinic semialdehyde dehydrogenase SSADH. During the conversion from GABA to succinic semialdehyde by GABA transaminase, an amino group is removed from GABA and added to alpha-ketoglutarate from the Krebs cycle, thus providing an equal amount of glutamate for each molecule of GABA converted to succinic semialdehyde ("GABA shunt") [Hassel et al., 1998]. Alternatively, succinic semialdehydecan be converted to $\mathrm{GHB}$ by succinic semialdehyde reductase [Maitre, 1997; Kelly et al., 2002; Gibson et al., 2003]. In SSADH deficiency the final step of the GABA degradation pathway is shifted towards the production of the alternative by-product GHB [Gropman, 2003]. GHB has effects on multiple neurotransmitter systems, such as dopamine, serotonine, acetylcholine and GABA [Gibson et al., 2003]. High levels of GHB and GABA desensitize postsynaptic $\mathrm{GABA}(\mathrm{B})$ receptors resulting in a decrease in $\mathrm{G}$-protein coupled inwardly rectifying potassium (GIRK) channel function, which is believed likely to cause the seizures in affected individuals and the $A L D H 5 A 1^{-/-}$mouse model [Vardya et al., 2010].

The clinical phenotype of SSADH deficiency has a high intra- as well as interfamilial variability ranging from mild delayed intellectual, motor, speech and language development to severe neurological defects including seizures, hypotonia, ataxia and behavioral problems [Jakobs et al., 1993; Gibson et al., 1997; Pearl et al., 2003]. Therapeutic concepts in human and murine SSADH deficiency have been reviewed [Knerr et al., 2007; Kim et al., 2011]. Recently seizures were treated successfully with high-dose phenobarbital in a young SSADH deficiency patient [Yamakawa et al., 2012]. The GABA(B) receptor antagonist SGS-742 showed positive effects in a Phase II double-blind, placebo-controlled clinical trial in patients with mild cognitive impairment [Froestl et al., 2004]. Pearl et al. [2009] could show that SGS-742 significantly improves the spike-wave duration in a dose dependent manner and also controls absence seizures.

Even though approximately 50 mutations in the SSADH encoding aldehyde dehydrogenase 5al gene (ALDH5A1; OMIM 610045) are associated with SSADH deficiency to date (HGMD Professional 2012.1; for a recent review on disease causing mutations in ALDH5A1 see Kim et al. [2011]), only one has so far been reported to affect $\mathrm{NAD}^{+}$binding.

Here, we present now a new homozygous missense mutation, c.901A $>$ G (p.K301E), in $A L D H 5 A 1$ and provide experimental evidence that this change causes SSADH deficiency in affected individuals, most probably owing to disturbed $\mathrm{NAD}^{+}$binding and molecular destabilization of the gene product, as shown by protein modeling.

\section{PATIENTS AND METHODS}

\section{Patients}

This study was carried out in accordance with the ethical standards of the appropriate national and institutional committees. An
Iranian family with severe ID and a history of generalized tonicclonic seizures without hyperactivity or attention deficit was ascertained and written informed consent obtained from the parents. The family pedigree and facial aspects of affected individuals are shown in Figure 1A,B respectively. All affected individuals were born full-term after uneventful pregnancies with birth weights between 3,000 and 3,500 g. There is a history of hypotonia during the neonatal period in this family. Nystagmus was not observed in any patient, but there is a history of unilateral glaucoma in V-9, which has started at the age of 11 years. All affected members had developmental delay and speech delay (4-6 years), walking started at 3.5 years of age. The index patient $\mathrm{V}-11$ suffers from severe ID (IQ 35) like both his affected sisters (V-9, IQ 30, and V-12, IQ 35) (Table I).

Apart from epilepsy, the patients suffered from no other neurologic disorders, and they had no neuropsychiatric problems. Seizures were successfully treated with carbamazepine. Brain MRI scans or metabolic tests were not performed in this family.

The ethnically matched control panel included 94 unrelated healthy individuals. Additionally, 124 unrelated healthy German individuals were also directly screened for the mutation. Samples from patients as well as from controls were obtained after receiving informed consent. Moreover, we used the publically available sequencing data from the Exome Variant Server (NHLBI Exome Sequencing Project (ESP), Seattle, WA, URL: http://evs.gs. washington.edu/EVS/), 200 Danish individuals [Li et al., 2010] and 185 genomes of healthy individuals made available by the 1,000 Genome Project (URL: http://www.1000genomes.org/) to further extend the control cohort.

\section{SNP Array}

DNA was extracted from peripheral blood of the patients (V-9, V-11, and $\mathrm{V}-12$ ), one healthy sibling (V-2), their parents (IV-3 and IV-4) and their affected cousin (V-1) using standard procedures. Karyotype analysis by G-banding was performed. The karyotype of all patients was normal and fragile X-syndrome was excluded. Genotyping (SNP analysis) was performed using the Human 610-Quad BeadChip (Illumina) following the protocol of the manufacturer. Linkage analysis was performed using the Merlin software [Abecasis et al., 2002]. Details of data quality controls and linkage analysis have been published elsewhere [Garshasbi et al., 2006].

\section{Mutation Screening and Evaluation}

Mutation screening of ALDH5A1 exons and exon-intron boundaries was performed in the index patient in 11 independent PCR reactions from genomic DNA (Table SI). We employed the PyMOL software (The PyMOL Molecular Graphics System, Version 1.5.0.1 Schrödinger, LLC) to model the ALDH5A1 protein structure based on the wildtype crystal structure (PDB 2w8o) available in the Protein Data Bank PDB [Berman et al., 2000] and performed an in silico analysis to estimate the effect of p.K301E on protein stability using Concoord/PBSA [Benedix et al., 2009; Potapov et al., 2009]. In silico prediction of pathogenic effects of p.K301E were performed with SIFT [Ng and Henikoff, 2001], PolyPhen2 [Adzhubei et al., 2010], Mutation Taster [Schwarz et al., 2010], and 


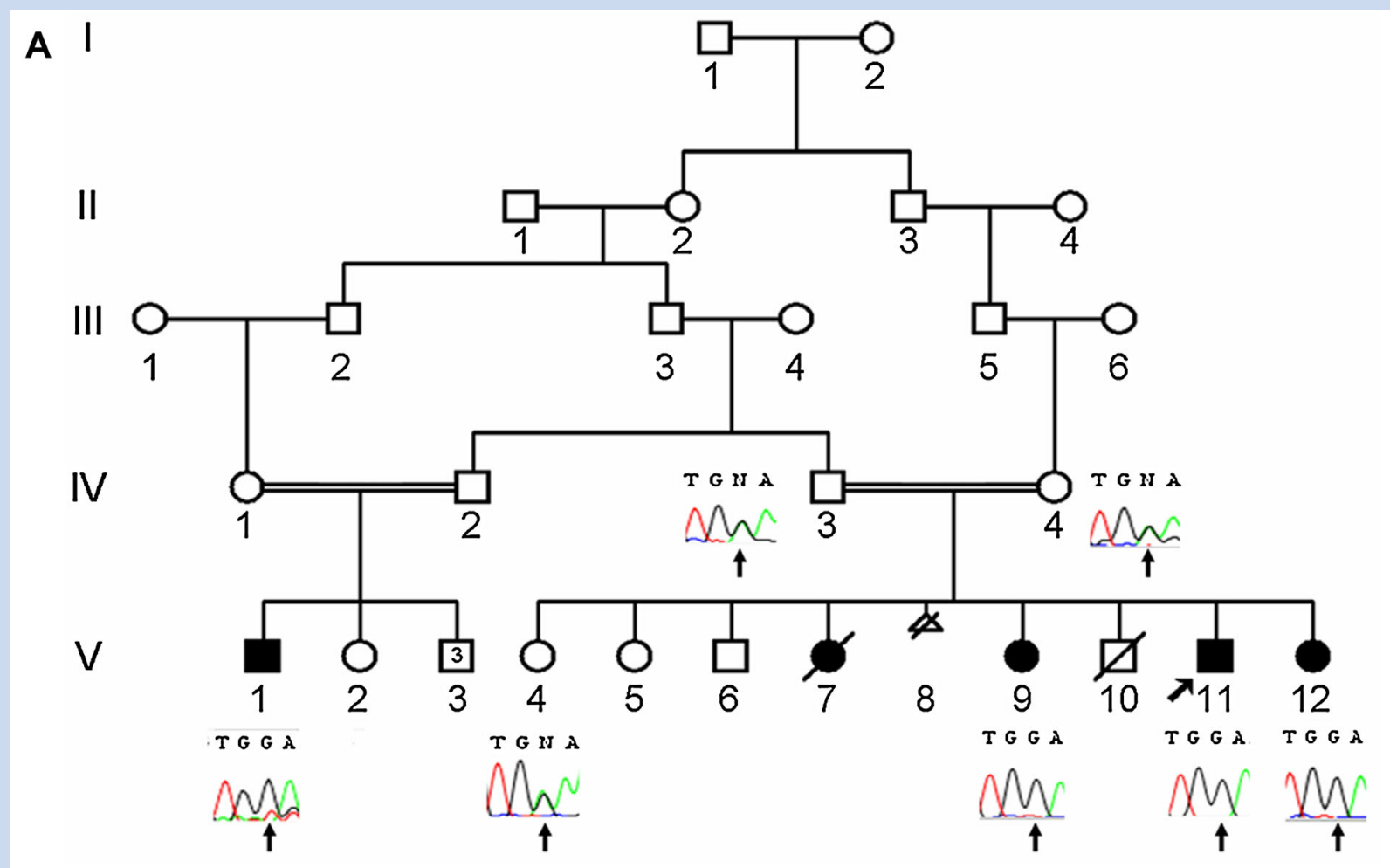

B
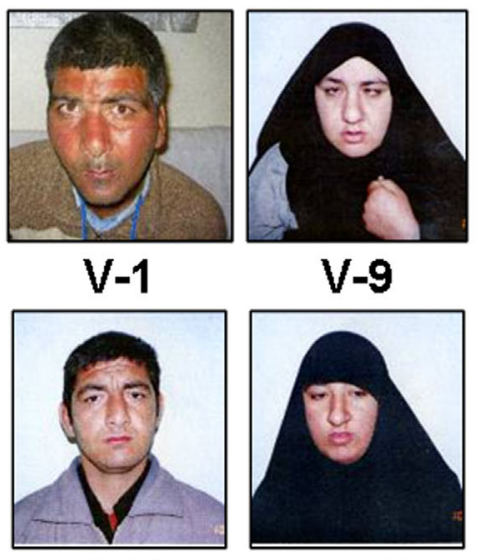

$\mathrm{V}-11$
$\mathrm{V}-9$

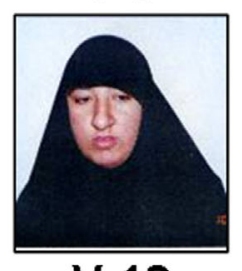

$\mathrm{V}-12$

C

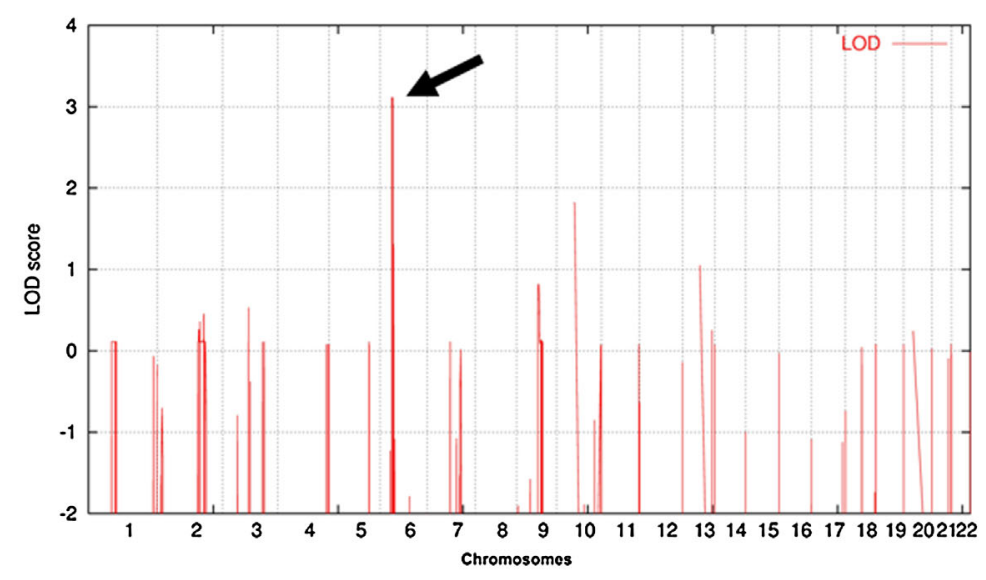

FIG. 1. A: Pedigree of the family $\mathbf{8 6 0 0 4 8 5}$. Full symbols denote affected individuals with ID. Sequence chromatograms next to all examined individuals show c.901A $>G$ in ALDH5A1 in a heterozygous (IV:3, IV:4, and V:4] or homozygous [V:1, V:9, V:11, and V:12] state. B: Affected individuals with SSADH deficiency. V-11 is the index patient. C: Result of whole genome parametric linkage analysis (Merlin software), showing a linkage interval with significant LOD score of 3.1 on chr.6p22-p21.32 (arrow).

Panther Classification System [Thomas et al., 2003; Thomas and Kejariwal, 2004] software tools.

\section{Enzymatic Assay}

An EBV immortalized lymphoblastoid cell line was established from the blood of the index patient $(\mathrm{V}-11)$ using standard procedures. Cells derived from 6 age and sex matched controls as well as the index patient were used to measure the level of SSADH enzyme activity. The method described by Gibson et al. [1991], based on the fluorometric assay by Cash et al. [1977], was modified for measuring NADH fluorescence in a microplate reader.

Cell pellets $\left(4 \times 10^{7}\right.$ cells $\left./ \mathrm{ml}\right)$ were resuspended in $100 \mathrm{mM}$ Tris$\mathrm{HCl}(\mathrm{pH}$ 8.6) and lysed by sonication twice for 2 cycles of $10 \mathrm{sec}$ at maximum power with a pause between the bursts. The final assay 
TABLE I. Features of Affected Individuals in Family 8600485

\begin{tabular}{|c|c|c|c|c|c|}
\hline Affected individual & Sex & Age at examination & 10 & OFC $[\mathrm{cm}]$ & Height $[\mathrm{cm}]$ \\
\hline V-1 & Male & 27 & $-{ }^{a}$ & 55 & 181 \\
\hline V-9 & Female & 27 & 30 & 51.5 & 163 \\
\hline V-11 & Male & 23 & 35 & 55.5 & 181 \\
\hline V-12 & Female & 21 & 35 & 52 & 157 \\
\hline
\end{tabular}

$\mathrm{OFC}$, occipitofrontal circumference.

${ }^{a}$ Diagnosed as severely intellectually disabled without 10 testing.

contained $100 \mu \mathrm{l}$ cell lysate (approxiamtely $0.6-0.7 \mathrm{mg}$ of total protein). SSADH activity was assayed for $30 \mathrm{~min}$ at $37^{\circ} \mathrm{C}$ in a final volume of $300 \mu \mathrm{l}$ in buffer containing $100 \mathrm{mM}$ Tris-HCl (pH 8.6), $0.1 \mathrm{mM}$ EDTA, $20 \mathrm{mM} \beta$-mercaptoethanol, $50 \mathrm{mM} \mathrm{KCl}$ and $0.1 \%$ Triton-X $100(\mathrm{v} / \mathrm{v}), 3 \mathrm{mM}$ succinic semialdehyde (Santa Cruz Biotechnology, Inc., Heidelberg, Germany) and NAD ${ }^{+}$ (Sigma-Aldrich Chemie GmbH, Taufkirchen bei München, Germany) in concentrations ranging from 0.01 to $2 \mathrm{mM}$. Succinic semialdehyde (Santa Cruz Biotechnology) was used at a concentration of $3 \mathrm{mM}$. Blank reactions contained water substituted for succinic semialdehyde. The reaction was stopped by heating for $5 \mathrm{~min}$ at $100^{\circ} \mathrm{C}$. After cooling down on ice for $10 \mathrm{~min}$, probes were centrifuged at $4^{\circ} \mathrm{C}$ $(20,800 \mathrm{~g})$ to remove cell debris. NADH fluorescence was measured in a POLARstar Omega microplate reader (BMG LABTECH GmbH, Ortenberg, Germany; excitation $355 \mathrm{~nm}$, emission $460 \mathrm{~nm}$ ).

\section{RESULTS}

\section{Linkage Analysis and Mutation Screening}

Parametric linkage analysis including individuals from both branches of family 8600485 (IV-3, IV-4, V-1, V-4, V-9, V-11, and V12) revealed a single interval of homozygosity on chromosome 6p22-p21.32 with a significant LOD score of 3.1 (Fig. 1C). The linkage interval had a size of $9.67 \mathrm{Mb}$ and was flanked by the heterozygous SNPs rs9379512 (chr6: 23499628, UCSC genome browser March 2006 assembly) and rs10484569 (chr6: 33166930, UCSC genome browser March 2006 assembly). The interval contains 284 genes including ALDH5A1. As the patient phenotype was strongly suggestive of SSADH deficiency, we screened ALDH5A1 for mutations and found an $A>G$ substitution (c.901A $>G$ ) in exon 6 (NM_001080) resulting in a change from leucine to glutamic acid at amino acid position 301 (p.K301E; NP_001071) (Fig. 2A). This missense mutation co-segregated with the disease in the core family (Fig. 1A) and was also present in the affected cousin. c.901A $>\mathrm{G}$ was absent from the control panel as well as from the 185 healthy individuals studied by the 1,000 Genomes Project and the exomes from 200 Danish individuals and is not listed in the Exome Variant Server (ESP6500).

The affected nucleotide c.901A has a PhyloP score of 5.031 and the lysine at the corresponding amino acid position 301 is highly conserved throughout the animal kingdom (eFig. S1 in Supporting information online). Taken together this evidence suggested strongly that c.901A $>\mathrm{G}$ had disease-causing potential. In order to further corroborate this hypothesis we analyzed the effect of the amino acid change caused by the mutation in silico and found that all four programes we used unanimously predicted deleterious consequences for SSADH function.

\section{In Silico Modeling of SSADH p.K301E}

To gain insights into the structural consequence of the p.K301E mutation in SSADH, we performed structure-based in silico modeling of the mutant protein. In the wild type structure, Lys301 connects two alpha helices and one beta strand, which are involved in the binding of $\mathrm{NAD}^{+}$(Fig. 2B, eFig. S2 in Supporting information online). The non-conservative change from a positively charged lysine to the negatively charged glutamine (Fig. 2C) likely destabilizes this area of the protein core. We have quantified this effect by performing in silico energy calculations with the Concoord/PBSA method [Benedix et al., 2009], which calculates stability changes upon mutation. The simulation revealed a decrease in stability of $6.67 \mathrm{kcal} / \mathrm{mol}$, which is more than the overall stability of most proteins [Dill, 1990]. Thus we hypothesized that the p.K301E mutation would severely reduce or even abolish SSADH activity.

\section{SSADH Activity Measurement}

Therefore, we next measured lymphoblast SSADH activity (blood, liquor, and urine were not available) in 6 age and sex matched controls and the index patient (V-11). Measurement of SSADH activity was performed in triplicate for each $\mathrm{NAD}^{+}$concentration on two consecutive days, respectively. We found SSADH activity to increase relating to elevated amounts of $\mathrm{NAD}^{+}$in the range of $0.01-0.1 \mathrm{mM}$ in the control cell lines, whereas in the patient cell line no SSADH activity could be detected (Fig. 3). At even higher NAD ${ }^{+}$ concentrations $(0.5-2 \mathrm{mM})$, SSADH was found to be still inactive in the patient cell line, whereas in the control cell lines a dose dependent decrease in SSADH activity was observed (not shown). Day-to-day variability in our study was in line with assay variability published by others [e.g., Gibson et al., 1991].

\section{DISCUSSION}

We present a novel missense mutation that is associated with SSADH deficiency in an Iranian family and leads to undetectable levels of SSADH activity. The affected individuals are from a large consanguineous family and present with developmental delay as 


\section{A SSADH}
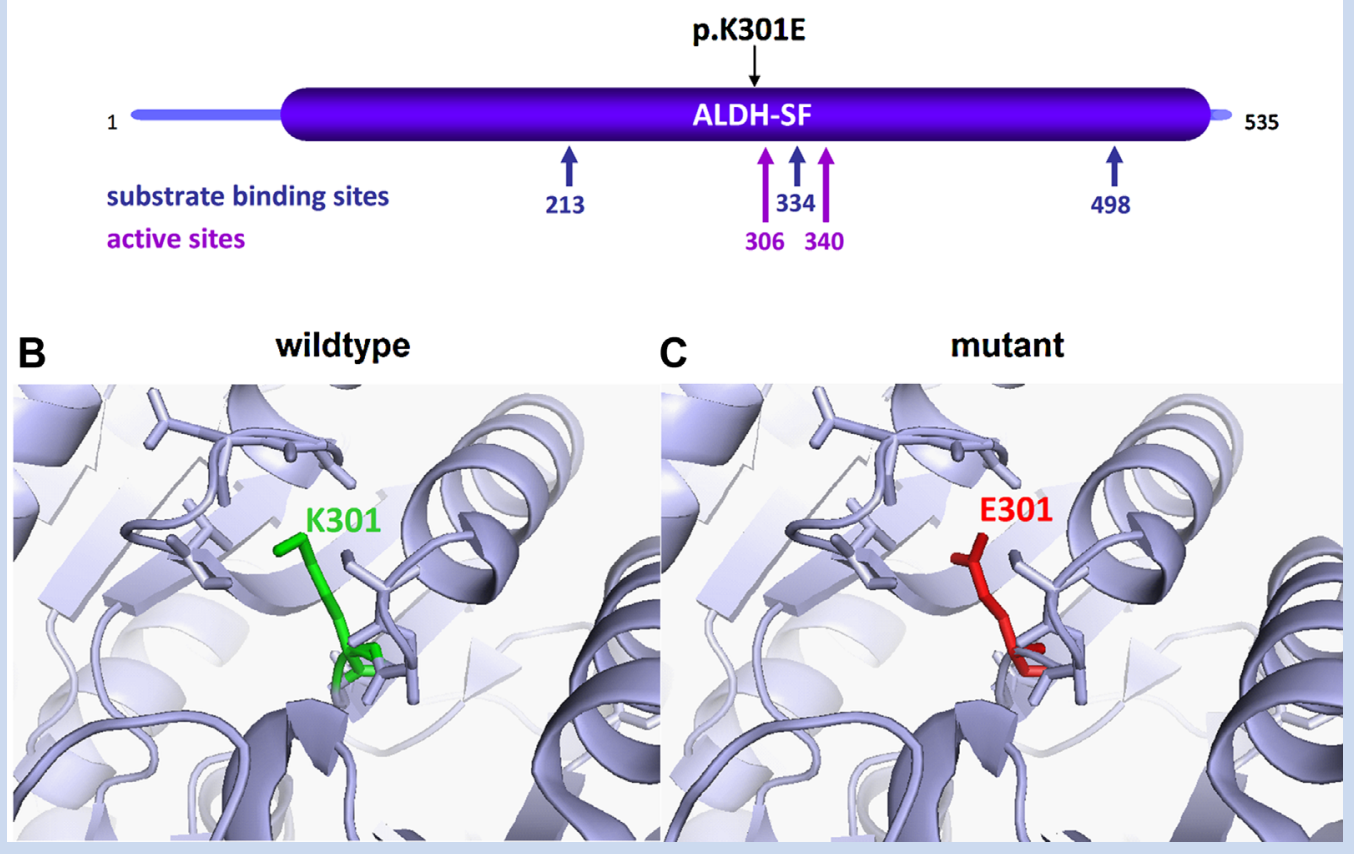

FIG. 2. A: Schematic representation of the ALDH5A1 gene product indicating the position of the change from lysine to glutamic acid at position 301 as well as the positions of the residues of the active sites and substrate binding sites. B: The residue affected by the missense mutation is presented as stick model with green color in the wildtype and red color in the mutant protein (C) respectively.

well as speech delay, severe ID, tonic-clonic seizures, and hypotonia during the neonatal period.

SSADH deficiency is a rare autosomal recessive disorder that has been reported in no more than about 450 affected individuals to

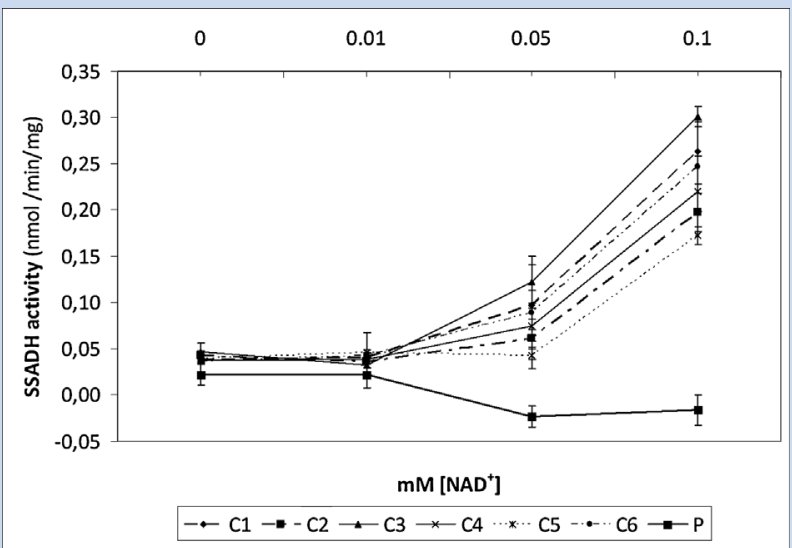

FIG. 3. Lymphoblast SSADH activity in cell lines of the affected individual and 6 controls at different $\mathrm{NAD}^{+}$concentrations $[0-0.1 \mathrm{mM}$ ]. SSADH activity is given as $\mathrm{nmol} / \mathrm{min} / \mathrm{mg}$ protein. Data were normalized against background activity. Each data point is the mean value of two separate experiments that were each performed in triplicate. Error bars represent the standard deviation. date [Pearl et al., 2011]. Parental consanguinity is observed in approximately $40 \%$ of the cases [Pearl et al., 2003]. The SSADH deficient patient database maintained by the Department of Neurology at Children's Medical Center in Washington DC is based on systematic questionnaire data of 60 patients. These data suggest that developmental delay, as well as ID are global findings in affected individuals (100\%), and that hypotonia (82\%), ataxia (77\%) as well as tonic-clonic seizures (53\%) are common clinical symptoms [Pearl et al., 2009, 2011]. All of these features, except for ataxia, are present in the affected individuals in our study and are consistent with the typical characteristics of human SSADH deficiency (as reviewed, e.g., by Kim et al., 2011; Pearl et al., 2011; or Knerr et al., 2008). Accompanying neuropsychiatric symptoms such as, for example, inattention, anxiety, aggressive behavior or autistic features, which occur in $72 \%, 40 \%, 21 \%$ or $12 \%$ of cases respectively [Kim et al., 2011; Pearl et al., 2011], were not obvious in our patients.

The murine knockout model $\left(A L D H 5 A 1^{-/-}\right)$is a viable phenocopy of the human SSADH deficiency and presents the most severe outcome of this disease. The clinical symptoms of the knockout mice comprise progressive ataxia, seizures and failure to thrive. Around postnatal Day 14 absence seizures appear, followed by tonic-clonic seizures around postnatal Day 20 and status epilepticus leading to rapid death during the fourth postnatal week [Hogema et al., 2001; Cortez et al., 2004; Gupta et al., 2004]. The substrate of SSADH, succinic semialdehyde, is derived from GABA after reuptake from the synaptic cleft into the presynaptic neuron. SSADH oxidizes succinic semialdehyde to succinate and 
simultaneously reduces the coenzyme $\mathrm{NAD}^{+}$to NADH. The Lysine affected by the p.K301E mutation we describe here is located in close spatial neighborhood to a glutamine at position 306 and a cysteine at position 340, which together constitute the active site environment. Under oxidized conditions SSADH is inactive and a disulfide bond is formed between the catalytic residues p.C340 and p.C342. A catalytic loop including residues 334-344 blocks the binding sites for succinic semialdehyde as well as for $\mathrm{NAD}^{+}$[Kim et al., 2009]. In silico analysis provided evidence that p.K301 is part of the protein core and involved in binding of $\mathrm{NAD}^{+}$and that p.K301E might lead to a destabilization of SSADH. This led us to the hypothesis that p.K301E severely reduces SSADH activity either by loss of protein stability through misfolding and subsequent degradation or, as the affected p.K301 connects two alpha helices and one beta strand that are involved in the binding of $\mathrm{NAD}^{+}$, by reduced $\mathrm{NAD}^{+}$binding capacity.

Loss of SSADH activity leads to accumulation of succinic semialdehyde, which is degraded to GHB by NADPH dependent aldoketo reductase 7A2 (AKR7A2) [Lyon et al., 2007]. GHB naturally occurs in the mammalian brain at a level of $<1 \%$ of its parent neurotransmitter GABA [Doherty et al., 1978] and acts on specific GHB receptors [Snead, 2000; Wu et al., 2004] as well as the GABA (B) receptor [Gervasi et al., 2003]. In the CSF of affected individuals, GHB levels are increased 65- to 230-fold, GABA levels are threefold elevated and glutamine is decreased [Gibson et al., 2003]. Comparable to affected humans; $A L D H 5 A 1^{-/-}$mice exhibit elevated levels of GABA and GHB in the brain [Hogema et al., 2001; Jansen et al., 2008]. In human and murine SSADH deficiency it could be observed that absence seizures are related to excessive GHB and GABA(B) mediated activity and that generalized convulsive seizures might be caused by overuse-dependent downregulation of GABA(A) and GABA(B) receptor activity [Buzzi et al., 2006; Wu et al., 2006; Pearl et al., 2011].

The assay we performed to test SSADH enzyme activity in the lymphoblastoid cell line of one affected individual proved our hypothesis to be true, as no SSADH activity could be detected. This is also in line with the results of Akaboshi et al. [2003] who assayed the activity of 27 disease-causing missense mutations observed in affected individuals. Five missense mutations (p.G176R, p.G268E, p.N335K, p.G409D and p.G533R) showed a nearly abolished enzyme activity $(<1-1 \%)$. While three of these mutations probably affect protein stability (p.G409D), or stability and oligomerization (p.G176R and p.G533R), p.G286E and p. $\mathrm{N} 335 \mathrm{~K}$ affect the catalytic function of SSADH. The protein residue p.N335 is located on the "dynamic catalytic loop" and probably leads to a severe distortion of the active site environment or reduced dynamics of the "catalytic loop" or both [Kim et al., 2009]. However, only p.G268 seems to be involved in binding of $\mathrm{NAD}^{+}$, being one of the residues creating the binding pocket for the adenine base of $\mathrm{NAD}^{+}$. Thus p.G268E might lead to a loss of $\mathrm{NAD}^{+}$binding ability and consequently to a loss of SSADH activity [Kim et al., 2009]. To our knowledge the p.K301E mutation we present here is now only the second missense mutation with a potential effect on the binding of $\mathrm{NAD}^{+}$that results in undetectable SSADH activity. In controls, SSADH activity increased using $\mathrm{NAD}^{+}$ ranging from 0.01 to $0.1 \mathrm{mM} \mathrm{NAD}^{+}$. Higher $\mathrm{NAD}^{+}$concentrations led to a decrease of enzyme activity in these samples. This could be explained by the fact that during oxidation of succinic semialdehyde to succinate, $\mathrm{NAD}^{+}$is reduced to $\mathrm{NADH}$, which is known to exhibit an inhibitory effect for several mammalian SSADHs [Duncan and Tipton, 1971; Blaner and Churchich, 1979; Rivett and Tipton, 1981; Kang et al., 2005]. Moreover, as a consequence of this, succinic semialdehyde is accumulated leading in turn to substrate inhibition of SSADH [e.g., Kammerat and Veldstra, 1968; Blaner and Churchich, 1979].

In conclusion, our study identified a novel homozygous missense mutation in ALDH5A1 that is associated with SSADH deficiency and severe ID. The mutation is located near the active site and is thus only the second mutation identified to date that might not necessarily cause a loss of SSADH activity solely through protein misfolding and subsequent degradation, but rather affect SSADH activity through an impairment of $\mathrm{NAD}^{+}$binding.

\section{ACKNOWLEDGMENTS}

We are very grateful to the affected individuals and their family for their participation in the study. We thank Susanne Freier and Sabine Otto for expert technical assistance and Mrs. Sanaz Arzhangi for her contribution in obtaining patient information. H.H.R., L. M., H.N., and K.K. participate in the GENCODYS Consortium.

\section{REFERENCES}

Abecasis GR, Cherny SS, Cookson WO, Cardon LR. 2002. Merlinrapid analysis of dense genetic maps using sparse gene flow trees. Nat Genet 30:97-101.

Akaboshi S, Hogema BM, Novelletto A, Malaspina P, Salomons GS, Maropoulos GD, Jakobs C, Grompe M, Gibson KM. 2003. Mutational spectrum of the succinate semialdehyde dehydrogenase (ALDH5A1) gene and functional analysis of 27 novel disease-causing mutations in patients with SSADH deficiency. Hum Mutat 22:442-450.

Adzhubei IA, Schmidt S, Peshkin L, Ramensky VE, Gerasimova A, Bork P, Kondrashov AS, Sunyaev SR. 2010. A method and server for predicting damaging missense mutations. Nat Methods 7:248-249.

Benedix A, Becker CM, de Groot BL, Caflisch A, Böckmann RA. 2009. Predicting free energy changes using structural ensembles. Nat Methods 6:3-4.

Berman HM, Westbrook J, Feng Z, Gilliland G, Bhat TN, Weissig H, Shindyalov IN, Bourne PE. 2000. The protein data bank. Nucleic Acids Res 28:235-242.

Blaner WS, Churchich J. 1979. Succinic semialdehyde dehydrogenase reactivity of lysyl residues. J Biol Chem 254:1794-1798.

Buzzi A, Wu Y, Frantseva MV, Perez Velazquez JL, Cortez MA, Liu CC, Shen LQ, Gibson KM, Snead OC III. 2006. Succinic semialdehyde dehydrogenase deficiency: GABA B receptor-mediated function. Brain Res 1090:15-22.

Cash C, Ciesielski L, Maitre M, Mandel P. 1977. Purification and properties of rat brain succinic semialdehyde dehydrogenase. Biochimie 59:257-268.

Cortez MA, Wu Y, Gibson KM, Snead OC III. 2004. Absence seizures in succinic semialdehyde dehydrogenase deficient mice: A model of juvenile absence epilepsy. Pharmacol Biochem Behav 79:547-553.

Dill KA. 1990. Dominant forces in protein folding. Biochemistry 29:7133-7155.

Doherty JD, Hattox SE, Snead OC, Roth RH. 1978. Identification of endogenous gamma-hydroxybutyrate in human and bovine brain and 
its regional distribution in human, guinea pig and rhesus monkey brain. J Pharmacol Exp Ther 207:130-139.

Duncan RJS, Tipton KF. 1971. The kinetics of pig brain aldehyde dehydrogenase. Eur J Biochem 22:538-543.

Exome Variant Server, NHLBI. Exome Sequencing Project (ESP), Seattle, WA. URL: http://evs.gs.washington.edu/EVS/ [May, 2012].

Froestl W, Gallagher M, Jenkins H, Madrid A, Melcher T, Teichman S, Mondadori CG, Pearlman R. 2004. SG S742: The first GABA(B) receptor antagonist in clinical trials. Biochem Pharmacol 68:1479-1487.

Garshasbi M, Motazacker MM, Kahrizi K, Behjati F, Abedini SS, Nieh SE, Firouzabadi SG, Becker C, Rüschendorf F, Nürnberg P, Tzschach A, Vazifehmand R, Erdogan F, Ullmann R, Lenzner S, Kuss AW, Ropers HH, Najmabadi H. 2006. SNP array-based homozygosity mapping reveals $\mathrm{MCPH} 1$ deletion in family with autosomal recessive mental retardation and mild microcephaly. Hum Genet 118:708-715.

Gervasi N, Monnier Z, Vincent P, Paupardin-Tritsch D, Hughes SW, Crunelli V, Leresche N. 2003. Pathway-specific action of gamma-hydroxybutyric acid in sensory thalamus and its relevance to absence seizures. J Neurosci 23:11469-11478.

Gibson KM, Lee CF, Chambliss KL, Kamali V, Francois B, Jaeken J, Jakobs C. 1991. 4-Hydroxybutyric, aciduria: Application of a fluorometric assay to the determination of succinic semialdehyde dehydrogenase activity in extracts of cultured human lymphoblasts. Clin Chim Acta 196:219-221.

Gibson KM, Christensen E, Jakobs C, Fowler B, Clarke MA, Hammersen G, Raab K, Kobori J, Moosa A, Vollmer B, Rossier E, Iafolla AK, Matern D, Brouwer OF, Finkelstein J, Aksu F, Weber HP, Bakkeren JA, Gabreels FJ, Bluestone D, Barron TF, Beauvais P, Rabier D, Santos C, Umansky R, Lehnert W. 1997. The clinical phenotype of succinic semialdehyde dehydrogenase deficiency (4-hydroxybutyric aciduria): Case reports of 23 new patients. Pediatrics 99:567-574.

Gibson KM, Hoffmann GF, Hodson AK, Bottiglieri T, Jakobs C. 1998. 4Hydroxybutyric acid and the clinical phenotype of succinic semialdehyde dehydrogenase deficiency, an inborn error of GABA metabolism. Neuropediatrics 29:14-22.

Gibson KM, Gupta M, Pearl PL, Tuchman M, Vezina LG, Snead OC III, Smit LM, Jakobs C. 2003. Significant behavioral disturbances in succinic semialdehyde dehydrogenase (SSADH) deficiency (gamma-hydroxybutyric aciduria). Biol Psychiatry 54:763-768.

Gropman A. 2003. Vigabatrin and newer interventions in succinic semialdehyde dehydrogenase deficiency. Ann Neurol 54:S66-72.

Gupta M, Polinsky M, Senephansiri H, Snead OC, Jansen EE, Jakobs C, Gibson KM. 2004. Seizure evolution and amino acid imbalances in murine succinate semialdehyde dehydrogenase (SSADH) deficiency. Neurobiol Dis 16:556-562.

Hassel B, Johannessen CU, Sonnewald U, Fonnum F. 1998. Quantification of the GABA shunt and the importance of the GABA shunt versus the 2oxoglutarate dehydrogenase pathway in GABAergic neurons. J Neurochem 71:1511-1518.

Hogema BM, Gupta M, Senephansiri H, Burlingame TG, Taylor M, Jakobs C, Schutgens RB, Froestl W, Snead OC, Diaz-Arrastia R, Bottiglieri T, Grompe M, Gibson KM. 2001. Pharmacologic rescue of lethal seizures in mice deficient in succinate semialdehyde dehydrogenase. Nat Genet 29:212-216

Jansen EE, Struys E, Jakobs C, Hager E, Snead OC, Gibson KM. 2008. Neurotransmitter alterations in embryonic succinate semialdehyde dehydrogenase (SSADH) deficiency suggest a heightened excitatory state during development. BMC Dev Biol 8:112.

Jakobs C, Jaeken J, Gibson KM. 1993. Inherited disorders of GABA metabolism. J Inherit Metab Dis 16:704-715.
Kammeraat C, Veldstra H. 1968. Characterization of succinate semialdehyde dehydrogenase from rat brain. Biochim Biophys Acta 151:1-10.

Kang JH, Park YB, Huh TL, Lee WH, Choi MS, Kwon OS. 2005. High-level expression and characterization of the recombinant enzyme, and tissue distribution of human succinic semialdehyde dehydrogenase. Protein Expr Purif 44:16-22.

Kelly VP, Sherratt PJ, Crouch DH, Hayes JD. 2002. Novel homodimeric and heterodimeric rat gamma-hydroxybutyrate synthases that associate with the golgi apparatus define a distinct subclass of aldo-keto reductase 7 family proteins. Biochem J 366:847-861.

Kim YG, Lee S, Kwon OS, Park SY, Lee SJ, Park BJ, Kim KJ. 2009. Redoxswitch modulation of human SSADH by dynamic catalytic loop. EMBO J 28:959-968.

Kim KJ, Pearl PL, Jensen K, Snead OC, Malaspina P, Jakobs C, Gibson KM. 2011. Succinic semialdehyde dehydrogenase: Biochemical-molecularclinical disease mechanisms, redox regulation, and functional significance. Antioxid Redox Signal 15:691-718.

Knerr I, Pearl PL, Bottiglieri T, Snead OC, Jakobs C, Gibson KM. 2007. Therapeutic concepts in succinate semialdehyde dehydrogenase (SSADH; ALDH5A1) deficiency (gamma-hydroxybutyric aciduria). Hypotheses evolved from 25 years of patient evaluation, studies in $A L D H 5 A 1^{-1-}$ mice and characterization of gamma-hydroxybutyric acid pharmacology. J Inherit Metab Dis 30:279-294.

Knerr I, Gibson KM, Jakobs C, Pearl PL. 2008. Neuropsychiatric morbidity in adolescent and adult succinic semialdehyde dehydrogenase deficiency patients. CNS Spectr 13:598-605.

Li Y, Vinckenbosch N, Tian G, Huerta-Sanchez E, Jiang T, Jiang H, Albrechtsen A, Andersen G, Cao H, Korneliussen T, Grarup N, Guo Y, Hellman I, Jin X, Li Q, Liu J, Liu X, Sparsø T, Tang M, Wu H, Wu R, Yu C, Zheng H, Astrup A, Bolund L, Holmkvist J, Jørgensen T, Kristiansen K, Schmitz O, Schwartz TW, Zhang X, Li R, Yang H, Wang J, Hansen T, Pedersen O, Nielsen R, Wang J. 2010. Resequencing of 200 human exomes identifies an excess of low-frequency non-synonymous coding variants. Nat Genet 42:969-972.

Lyon RC, Johnston SM, Watson DG, McGarvie G, Ellis EM. 2007. Synthesis and catabolism of gamma-hydroxybutyrate in SH-SY5Y human neuroblastoma cells: Role of the aldo-keto reductase AKR7A2. J Biol Chem 282:25986-25992.

Maitre M. 1997. The gamma-hydroxybutyrate signalling system in brain: Organization and functional implications. Prog Neurobiol 51:337-361.

Mason PE, Kerns WP II. 2002. Gamma hydroxybutyric acid (GHB) intoxication. Acad Emerg Med 9:730-739.

Ng PC, Henikoff S. 2001. Predicting deleterious amino acid substitutions. Genome Res 11:863-874.

Pearl PL, Gibson KM, Acosta MT, Vezina LG, Theodore WH, Rogawski MA, Novotny EJ, Gropman A, Conry JA, Berry GT, Tuchman M. 2003. Clinical spectrum of succinic semialdehyde dehydrogenase deficiency. Neurology 60:1413-1417.

Pearl PL, Gibson KM, Cortez MA, Wu Y, Carter Snead O III, Knerr I, Forester K, Pettiford JM, Jakobs C, Theodore WH. 2009. Succinic semialdehyde dehydrogenase deficiency: Lessons from mice and men. J Inherit Metab Dis 32:343-352.

Pearl PL, Shukla L, Theodore WH, Jakobs C, Michael Gibson K. 2011. Epilepsy in succinic semialdehyde dehydrogenase deficiency, a disorder of GABA metabolism. Brain Dev 33:796-805.

Potapov V, Cohen M, Schreiber G. 2009. Assessing computational methods for predicting protein stability upon mutation: Good on average but not in the details. Protein Eng Des Sel 22:553-560.

Rivett AJ, Tipton KF. 1981. Kinetic studies with rat-brain succinic-semialdehyde dehydrogenase. Eur J Biochem 117:187-193. 
Schwarz JM, Rödelsperger C, Schuelke M, Seelow D. 2010. Mutation taster evaluates disease-causing potential of sequence alterations. Nat Methods 7:575-576.

Snead OC III. 2000. Evidence for a G protein-coupled gamma-hydroxybutyric acid receptor. J Neurochem 75:1986-1996.

Thomas PD, Kejariwal A, Campbell MJ, Mi H, Diemer K, Guo N, Ladunga I, Ulitsky-Lazareva B, Muruganujan A, Rabkin S, Vandergriff JA, Doremieux O. 2003. PANTHER: A browsable database of gene products organized by biological function, using curated protein family and subfamily classification. Nucleic Acids Res 31:334-341.

Thomas PD, Kejariwal A. 2004. Coding single-nucleotide polymorphisms associated with complex versus Mendelian disease: Evolutionary evidence for differences in molecular effects. Proc Natl Acad Sci USA 101:15398-15403.

Vardya I, Drasbek KR, Gibson KM, Jensen K. 2010. Plasticity of postsynaptic, but not presynaptic, GABA B receptors in SSADH deficient mice. Exp Neurol 225:114-122.

Wu Y, Ali S, Ahmadian G, Liu CC, Wang YT, Gibson KM, Calver AR, Francis J, Pangalos MN, Carter Snead O III. 2004. Gamma-hydroxybutyric acid (GHB) and gamma-aminobutyric acid B receptor (GABABR) binding sites are distinctive from one another: Molecular evidence. Neuropharmacology 47:1146-1156.

Wu Y, Buzzi A, Frantseva M, Velazquez JP, Cortez M, Liu C, Shen L, Gibson KM, Snead OC III. 2006. Status epilepticus in mice deficient for succinate semialdehyde dehydrogenase: GABA A receptor-mediated mechanisms. Ann Neurol 59:42-52.

Yamakawa Y, Nakazawa T, Ishida A, Saito N, Komatsu M, Matsubara T, Obinata K, Hirose S, Okumura A, Shimizu T. 2012. A boy with a severe phenotype of succinic semialdehyde dehydrogenase deficiency. Brain Dev 34:107-112.

\section{SUPPORTING INFORMATION}

Additional supporting information may be found in the online version of this article at the publisher's web-site.

FIG. S1. Partial amino acid sequence alignment of the human SSADH protein and its homologues in the animal, plant and fungi kingdom. The position of the missense mutation is indicated by an arrow. The respective amino acid is identical in the 13 proteins.

FIG. S2. Overview of Lys301 (green) and $\mathrm{NAD}^{+}$binding site (yellow) in stick representation. The ADP moiety of a $\mathrm{NAD}^{+}$ molecule is shown in orange.

TABLE SI. Primers used for amplification of ALDH5A1. 